\title{
Data Assimilation in Brain Tumor Models
}

\author{
Joshua Mc Daniel $^{1}$, Eric Kostelich ${ }^{1}$, Yang Kuang ${ }^{1}$, John Nagy $^{2}$, Mark Preul ${ }^{3}$, Nina \\ Z. Moore $^{3}$, Nikolay Matirosyan ${ }^{3}$ \\ 1 School of Mathematical and Statistical Sciences, Arizona State University, Tempe, AZ \\ 85287, USA Joshua.McDaniel@asu.edu, kostelich@asu.edu, kuang@asu.edu \\ 2 Department of Biology, Scottsdale Community College, Scottsdale, AZ 85256-2626, USA \\ john.nagy@sccmail.maricopa.edu \\ 3 Department of Neurosurgery Research, Barrow Neurological Institute, St. Josephs \\ Hospital and Medical Center, Phoenix, AZ 85013, USA Mark.Preul@chw .edu
}

\section{Introduction}

A typical problem in applied mathematics and science is to estimate the future state of a dynamical system given its current state. One approach aimed at understanding one or more aspects determining the behavior of the system is mathematical modeling. This method frequently entails formulation of a set of equations, usually a system of partial or ordinary differential equations. Model parameters are then measured from experimental data or estimated from computer simulation or other methods, for example chi-squared parameter optimization as done in [23] or genetic algorithms which are frequently used in neuroscience [30]. Solutions to the model are then studied through mathematical analysis and numerical simulation usually for qualitative fit to the dynamical system of interest and any relative time-series data that is available. While mathematical modeling can provide meaningful insight, it may have limited predictive value due to idealized assumptions underlying the model, measurement error in experimental data and parameters, and chaotic behavior in the system. In this chapter we explore a different approach focused on optimal state estimation given a model and observational data of a biological process, while accounting for the relative uncertainty in both. The case explored here is the growth and spread of glioblastoma multiforme (GBM), a very aggressive form of glioma brain tumor which remains extremely difficult to manage clinically. The method employed is different from other approaches used in biology in that it is independent of the mathematical model and seeks an optimal initial condition. This is in contrast to other techniques such as those discussed in [17], which are model dependent and seek to find an optimal model parameterization given the observations and uncertainties in the system of interest.

The method discussed and implemented in this chapter is derived from numerical weather prediction in which initial conditions to atmospheric weather models must be updated frequently from noisy measurements. Chaos exhibited in the under- 


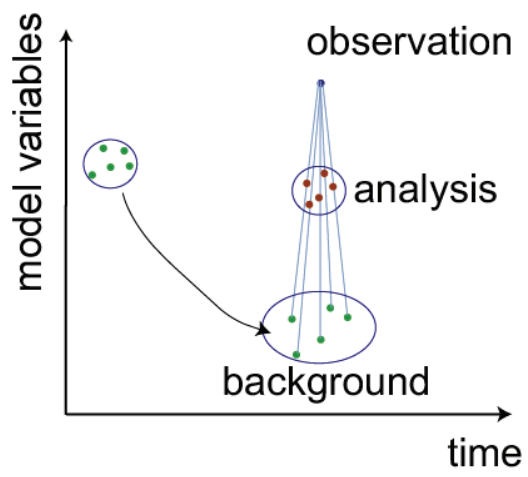

Fig. 1. Schematic illustration of the data assimilation procedure.

lying weather models leads to the propagation of uncertainty in the initial conditions over short time periods. Thus accurate forecasting requires update of forecast model initial conditions frequently (typically 6 hours in global models) based on current observational data. The algorithm for computing the update is known as data assimilation. Figure 1 summarizes the main elements of this procedure. One begins with a model trajectory that represents the best estimate of the true state of the system. This is advanced under the model, until a new observation becomes available, producing the background. An update called the analysis is computed and the process repeats. Under assumptions regarding the probability distribution of the background and observation error, the analysis is a maximum likelihood estimate of the true system state.

In this chapter we demonstrate how the data assimilation approach might be adapted to help anticipate the progression of clinical patient cases of GBM. GBM is chosen because the dynamics of tumor growth are dependent on the location and density of the underlying tumor cell population; hence, its dynamics are described by a spatiotemporal model. Also noisy observations in the form of magnetic resonance (MR) images are available which should provide insight into the current state of the tumor (meaning the cancer cell density at every point on the brain geometry). The overall approach is independent of the model; hence, this method may be used on a broad range of spatiotemporally complex biological systems.

There are two principal sources of uncertainty in estimating the future growth of GBM. First unlike the motions of the atmosphere, which can be described by well-established physical models, many details of the growth of glioblastoma tumor cells are poorly understood and stochastic in nature. Typically tumors exhibit genetic abnormalities leading to heterogeneous compositions of cells, metabolism, and vasculature. Brain anatomy varies on an individual basis, and tumors deform the brain geometry depending on their size and location (know as the mass effect).

The second problem arises from interpretation of MR imaging studies. Due to the risks associated with surgical treatment and the problem of accessing the tumor 


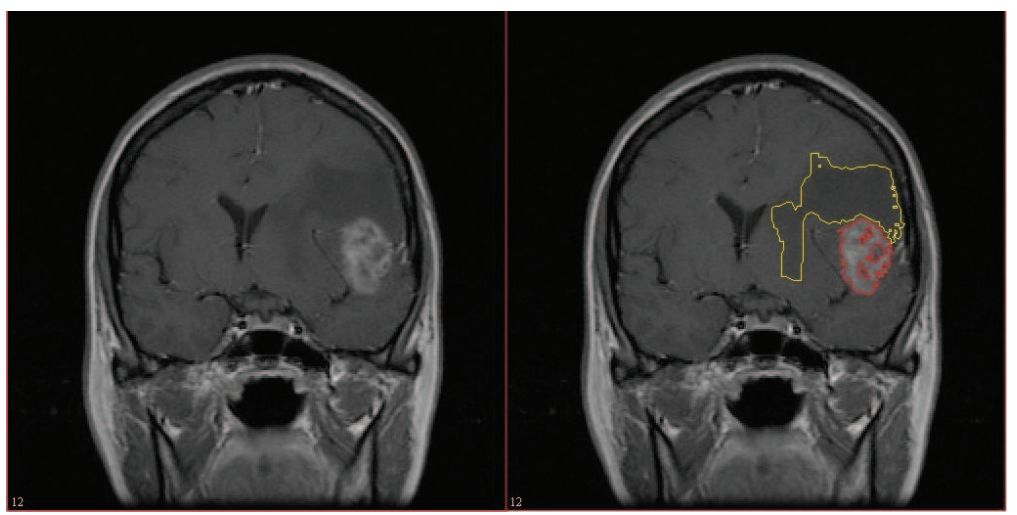

Fig. 2. A representative contrast enhanced magnetic resonance image of a GBM patient at initial diagnosis. The right panel shows the edema outlined in yellow and the enhancement region outlined in red. See text for additional details.

through the skull, noninvasive MR scans are the primary method for assessing the progression of gliomas, including GBM. MR scans are usually taken at intervals from weeks to months depending on the treatment regime. Patients are administered a contrast agent that highlights visibility of the tumor vasculature from the surrounding brain tissue. GBM tumors are highly vascular; thus, strength of contrast is typically interpreted as a measure of glioma cell density. The region of highest intensity is called the enhancing region and corresponds to area of highest blood vessel density, and most likely blood plasma that has leaked into surrounding tumor tissue. This area along with the nearby necrotic regions of less contrast are sometimes called the tumor core. The region of lower contrast, usually near the enhancing region, is called the edema, which is interpreted to be swelling of noncancerous brain tissue due to leaky and abnormal tumor vasculature and infiltration of "healthy" tissue by the tumor [8]. Figure 2 shows a contrast enhanced MR image of a patient tumor. The red and yellow boundaries mark the enhancing and edema regions respectively. The image also shows the mass effect of the tumor in the right hemisphere.

It is difficult to infer quantitative information about the underlying cell density from MRI data for many reasons. First the relationship (if any exists) between a pixel intensity at a given a point and the cell density there is unknown. Quantitative comparison of patient MRI data to a simulated tumor requires a common geometry. Thus a second problem, known as registration error, arises from inaccuracies in mapping the patient MR scan onto the associated brain atlas (model geometry) of the forecast model. Some sources of registration error are individual variance in patient brain geometry and the mass effect. Radiation necrosis resulting from treatment, which may appear similar to active tumor on the image, can further complicate MR image interpretation [22].

The goal of this chapter is to demonstrate the use of ensemble forecasting and data assimilation to make improved short-term (30-day) estimates of the growth and spread of a simulated brain tumor. The remainder of the chapter is organized as fol- 
lows. Section 2 provides a brief overview of two models of glioblastoma employed to simulate virtual brain tumors. Section 3 introduces the concept of ensemble forecasting and the history and rationale underlying this method. Section 4 introduces the mathematical aspects of data assimilation and presents a detailed derivation of both the basic Kalman filter for linear dynamical systems and a state-of-the-art extension to nonlinear problems known as the Local Ensemble Transform Kalman Filter (LETKF). Section 5 describes numerical experiments that demonstrate implementation of the LETKF on a brain tumor models where synthetic observations of a simulated tumor are generated. The results illustrate the potential feasibility of this approach to better forecast the evolution of individual patient lesions.

\section{Models of Glioblastoma}

Glioblastoma multiforme (GBM) is the most common primary malignant brain tumor, and its prognosis is very poor; patient survival is less than 15 months, on average, from initial diagnosis [20]. GBM tumors are highly aggressive, typically develop resistance to chemotherapy and radiotherapy [1], and can quickly invade large and sensitive regions of the brain, making complete surgical resection of the tumor impossible and post-surgical recurrence inevitable [4]. Because little clinical progress has been made against GBM, its biology remains the subject of intense study.

In this chapter we use two mathematical models to simulate tumor growth and expansion. These models are chosen because they each represent syntheses of some previous GBM modeling efforts and reflect increasing levels of complexity. Both models are simulated on a static but realistic brain geometry where diffusion rates are differentiated between white matter, gray matter, and other intracranial tissues. The first model, initially proposed by Swanson et. al. [24,25], considers a single class of glioma cells exhibiting exponential growth with cell motility governed by Fick's law. These assumptions yield the equation,

$$
\frac{\partial g}{\partial t}=\nabla \cdot(D(\mathbf{x}) \nabla g)+\alpha g .
$$

The diffusion rate of GBM cells is assumed to be faster in white matter than in gray. Each tissue type is further assumed to be homogeneous with respect to its diffusivity. Therefore, $D$ is taken to be piecewise constant within each tissue type, but may vary among types. Parameters in equation 1 can be estimated from in vitro studies, sequential patient MR studies, or the Einstein-Stokes relation [24].

Since the exponential growth term in equation 1 leads to unbounded tumor cell densities, a more realistic approach assumes that cells grow logistically with some carrying capacity, $T_{\max }$, at any given point in the model's domain. This modification gives [26]

$$
\frac{\partial g}{\partial t}=\nabla \cdot(D(\mathbf{x}) \nabla g)+\alpha g\left(1-\frac{g}{T_{\max }}\right) .
$$


We refer to equation 2 as the Logistic Swanson model. Baseline parameter values for this model are reported in table 1.

Table 1. Representative parameters for the logistic Swanson model, Eq. (2), in 2 dimensions.

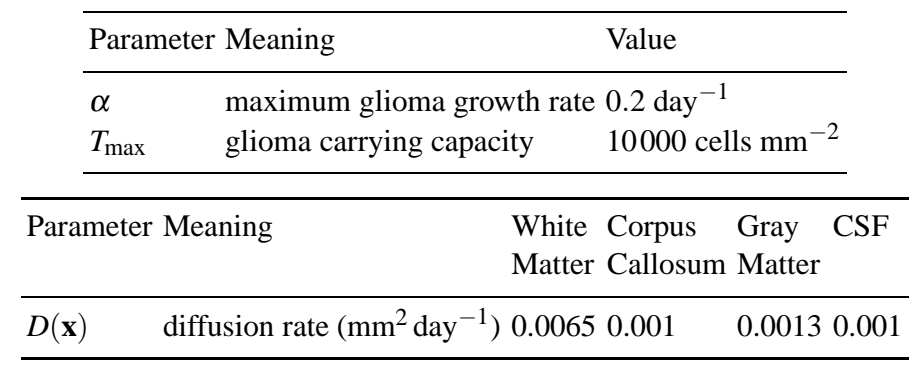

A more sophisticated modeling approach by Eikenberry et al. [5] considers two distinct phenotypic classes of tumor cells: proliferating and migrating. The proliferating cells grow logistically and produce a generalized chemorepellant which is assumed to induce transition to the migrating cell class at sufficiently high concentrations. Migrating cells degrade the extracellular matrix (ECM) and migrate away from the main tumor mass along the ECM gradient via haptotaxis. The transition between the two cell classes is stochastic. The four dependent model variables are

$$
\begin{aligned}
g(\mathbf{x}, t) & =\text { proliferating cell density } \\
m(\mathbf{x}, t) & =\text { migrating cell density } \\
c(\mathbf{x}, t) & =\text { chemorepellent density } \\
w(\mathbf{x}, t) & =\text { extracellular matrix }(\mathrm{ECM}) \text { density }
\end{aligned}
$$

and the Eikenberry model is expressed as a coupled system of four stochastic partial differential equations given by,

$$
\begin{aligned}
& \frac{\partial g}{\partial t}=\underbrace{\nabla \cdot\left(D_{G}(\mathbf{x}) \nabla g\right)}_{\text {diffusion }}-\underbrace{\nabla \cdot(\chi(\mathbf{x}) m \nabla w)}_{\text {haptotaxis }}+\underbrace{\alpha g\left(1-\frac{g+m}{T_{\max }}\right)}_{\text {logistic growth }}-\underbrace{\Phi}_{g-t o-m}+\underbrace{\Psi}_{m-t o-g} \\
& \frac{\partial m}{\partial t}=\underbrace{\nabla \cdot\left(D_{M}(\mathbf{x}) \nabla m\right)}_{\text {diffusion }}+\underbrace{\nabla \cdot(\chi(\mathbf{x}) m \nabla w)}_{\text {haptotaxis }}+\underbrace{\Phi}_{g-t o-m}-\underbrace{\Psi}_{m-t o-g}-\underbrace{\sigma(g, m)}_{\text {death }} \\
& \frac{\partial c}{\partial t}=\underbrace{D_{C} \nabla^{2} c}_{\text {diffusion }}+\underbrace{\gamma g}_{\text {production }}-\underbrace{\beta_{C} c}_{\text {degradation }} \\
& \frac{\partial w}{\partial t}=\underbrace{-\rho w\left(\frac{g+m}{\theta_{W}+T}\right)}_{\text {degradation }}+\underbrace{\alpha_{W} w(1-w)}_{\text {repair }}
\end{aligned}
$$


where $\Phi$ is the rate at which proliferating cells become migratory and $\Psi$ is the rate at which migratory cells become proliferating. Eikenberry et al. [5] assumed that the transition rate to the migratory phenotype is greatest when the cell density is low (such as at the edges of the tumor):

$$
\Phi=\Phi(g, m, c)=\tau g\left(\frac{\theta_{G}^{2}}{(g+m)^{2}+\theta_{G}^{2}}\right)\left(\frac{c}{\phi_{M}+c}\right) .
$$

The stochasticity arises from how $\Psi$ is implemented over the discretization of the model. The probability of transition from a migrating to a proliferating phenotype is assumed to be an exponential random variable with rate depending on the chemorepellent concentration,

$$
\lambda=\lambda_{0}\left(\frac{\theta_{G}}{\theta_{G}+c}\right) .
$$

Once $\lambda$ is determined, $\Psi$ is computed by finding the number of migrating cells that transition at each grid point and converting this to a cell density. At a given grid point the cell density must constitute at least one cell for any such transition to occur.

Cell death is assumed to result from crowding and occurs only when the cell density reaches a critical threshold, $M_{D}$ :

$$
\sigma(g, m)=\left\{\begin{array}{cc}
\left(\frac{g+m}{T_{\max }}\right)^{2}, & g+m>M_{D} \\
0, & \text { otherwise. }
\end{array}\right.
$$

Note the haptotaxis term in Eq. (3) is a modification of the original model and is included to preserve conservation of mass.

Table 2 displays the nominal parameter values for the Eikenberry model. The values used here differ slightly from those in [5] and were chosen so that the total tumor cell populations from both the Logistic Swanson model, Eq. (2), and the Eikenberry model grow at approximately the same rate. The diffusion rates, $D_{G}$ and $D_{M}$, in the Eikenberry model are smaller in regions corresponding to cerebrospinal fluid (CSF) than those in the Swanson model. See [5] for further details.

Both sets of equations are integrated using a brain geometry from the BrainWeb database, developed by the McConnel Brain Imaging Center of the Montreal Neurological Institute at McGill University [2]. We use the discrete anatomical model of a normal brain generated by McGill's MR simulator, which consists of a $181 \times 217 \times 181$ isotropic grid of $1 \mathrm{~mm}^{3}$ voxels in Talairach space [28]. Each voxel is classified as background, cerebro-spinal fluid (CSF), gray or white matter, fat, muscle/skin, skin, skull, or glial matter. The classification determines the boundary of the model domain, and the diffusion and haptotaxis coefficients at each grid point. To reduce the computational expense, the equations are integrated over a representative coronal slice at the center of the 3-dimensional domain, from which voxels representing the skull and similar tissue have been removed. The resulting 2-dimensional domain is a fixed $145 \times 143$ grid (the mass effect is not modeled). For simulation purposes, glial matter is treated as white matter, and the domain has 
Table 2. Representative parameters for the Eikenberry model, Eqs. (3-6), in 2 dimensions.

\begin{tabular}{|c|c|c|c|c|}
\hline \multicolumn{3}{|c|}{ Parameter Meaning } & \multicolumn{2}{|l|}{ value } \\
\hline$\alpha$ & maximum glioma growth rate & & 0.15 day $^{-}$ & \\
\hline$T_{\max }$ & glioma carrying capacity & & $10000 \mathrm{cel}$ & $1 \mathrm{~s} \mathrm{~mm}^{-2}$ \\
\hline$\alpha_{W}$ & maximum ECM recovery rate & & $0.01 \mathrm{day}^{-}$ & \\
\hline$\beta_{C}$ & chemorepellent degradation rate & & 0.25 day $^{-}$ & \\
\hline$D_{C}$ & chemorepellent diffusion rate & & $2.0 \mathrm{~mm}^{2} \mathrm{c}$ & day $^{-1}$ \\
\hline$\gamma$ & chemorepellent production rate & & $2.5 \times 10^{-}$ & 5 day $^{-1}$ \\
\hline$\lambda_{0}$ & maximum migrating to grow rate & & 2.5 day $^{-1}$ & \\
\hline$M_{D}$ & crowding death threshold & & 5000 cells & $\mathrm{mm}^{-2}$ \\
\hline$\phi_{M}$ & half-maximum grow to migrating coeff. & & $0.5 \mathrm{~mm}^{-2}$ & \\
\hline$\rho$ & maximum ECM remodeling rate & & 0.02 day $^{-}$ & \\
\hline$\tau$ & maximum grow to migrating transition rate & & 0.1 day $^{-1}$ & \\
\hline$\theta_{G}$ & half-maximum grow to migrating cell densi & & 100 cells 1 & $\mathrm{~mm}^{-2}$ \\
\hline$\theta_{W}$ & cell density at half-maximum ECM degrada & ation & 100 cells 1 & $\mathrm{~mm}^{-2}$ \\
\hline \multicolumn{2}{|c|}{ Parameter Meaning } & \multicolumn{3}{|c|}{ White Gray CSF } \\
\hline$D_{G}(\mathbf{x})$ & growing cell diffusion rate $\left(\mathrm{mm}^{2} \mathrm{day}^{-1}\right)$ & 0.02 & 0.004 & 0.0001 \\
\hline$D_{M}(\mathbf{x})$ & migrating cell diffusion rate $\left(\mathrm{mm}^{2}\right.$ day $\left.^{-1}\right)$ & 0.1 & 0.02 & 0.0001 \\
\hline$\chi(\mathbf{x})$ & haptotaxis coefficient $\left(\mathrm{mm}^{4}\right.$ day $\left.^{-1}\right)$ & 1.0 & 0.2 & 0 \\
\hline
\end{tabular}

been edited by hand to enumerate voxels comprising the corpus callosum (which is a favored migratory pathway for GBM cells [1]).

The spatial derivatives are approximated by finite differences, and the resulting set of ordinary differential equations is integrated using the second-order (in time) Heun's method with a fixed time step 0.5 day $^{-1}$ in the case of Logistic Swanson and a fourth-order Runge-Kutta method for the Eikenberry with fixed timestep of 0.1 day $^{-1}$. The numerical methods chosen here are for computational efficiency and haven't exhibited instabilities with the chosen timesteps. Given the discrete nature of the brain geometry, location-dependent parameters (such as the diffusion constants) are taken to be piecewise constant.

These models are chosen for this initial investigation because they are inexpensive to integrate, particularly in 2 dimensions, and are adequate for illustrating the basic ideas behind data assimilation, which is our principal focus here. Other efforts have modeled important aspects of GBM growth such as various forms of treatment [29], the mass effect of the tumor, and directed diffusion of GBM cells along white matter tracts [3]. Such refinements would undoubtedly be part of any data assimilation system intended for clinical application. 


\section{Ensemble Forecasting}

Ensemble forecasting, a technique used to assess and quantify the effect of uncertainty in a mathematical model of a dynamical system, was developed from early studies of chaotic behavior. A classic example, formulated by Edward Lorenz in 1963 [15], consisted of a system of three coupled ordinary differential equations modeling fluid flow. The system exhibited sensitive dependence on initial conditions. That is, small errors in non fixed-point initial conditions quickly propagated in time, leading to large differences in solutions. Even though trajectories had similar limit sets, they became uncorrelated over time even when the initial conditions were very similar. In the case of weather forecasting, like many other systems, one cannot sample the atmosphere at every point, observations are corrupted by noise, and any given weather model imperfect. This, coupled with chaotic behavior, leads to models that offer no predictive advantage over climatological averages. Even on time scales of a few days or less, uncertainties in the initial state of the atmosphere may lead to substantial forecast errors.

To account for uncertainties in a forecast model, Lorenz suggested in 1965 [16] that, instead of simulating a single initial condition under the model from a best estimate of the state of the atmosphere, one should evolve a set or ensemble of initial conditions, each from a statistically equivalent estimate of the true initial state. Then the ensemble gives a Monte Carlo estimate of the uncertainty in a given weather model. Under assumptions discussed in the next section, the ensemble mean constitutes an empirical maximum-likelihood estimate of the true state of the atmosphere. Ensemble forecasting became part of the routine operations at the U.S. and European weather centers in 1992 [14].

Figure 3 shows representative ensemble forecasts of geopotential height contours at $500 \mathrm{hPa}$. Each curve shows the result, from one initial condition on Oct. 12, 2010, of a forecast obtained by running the weather model for 3 days (top panels) and 7 days (bottom panels). Roughly speaking, the maps show the predicted locations where half the atmosphere's mass is above $5520 \mathrm{~m}$ (left panels) and $5760 \mathrm{~m}$ (right panels $)^{4}$.

Of greatest interest here is how the maps illustrate the magnitude of forecast uncertainty, which varies considerably in space as well as time. Because of the chaotic dynamics, the forecast uncertainty generally is larger at 7 days than at 3 days. The 5760-m contours (right panels) show considerable spread over the North Atlantic Ocean at 7 days, corresponding to especially large uncertainties in the forecast locations of the 500-hPa geopotential height.

In section 5 we illustrate the use of ensemble forecasting adapted to the two models of glioblastoma discussed in section 2. While both models exhibit simple, non-chaotic dynamics where the cancer cell population grows to carrying capacity

\footnotetext{
${ }^{4}$ The geopotential, $\Phi(z)$, is the work needed to raise a unit mass a vertical distance $z$ from mean sea level and accounts for the variation of the earth's gravitational field with latitude and elevation. The geopotential height is $\Phi(z) / g_{0}$, where $g_{0}=9.80665 \mathrm{~m} \mathrm{~s}^{-2}$ is the global average of gravitational acceleration at mean sea level. For more details, see Chapter 1 of [10].
} 

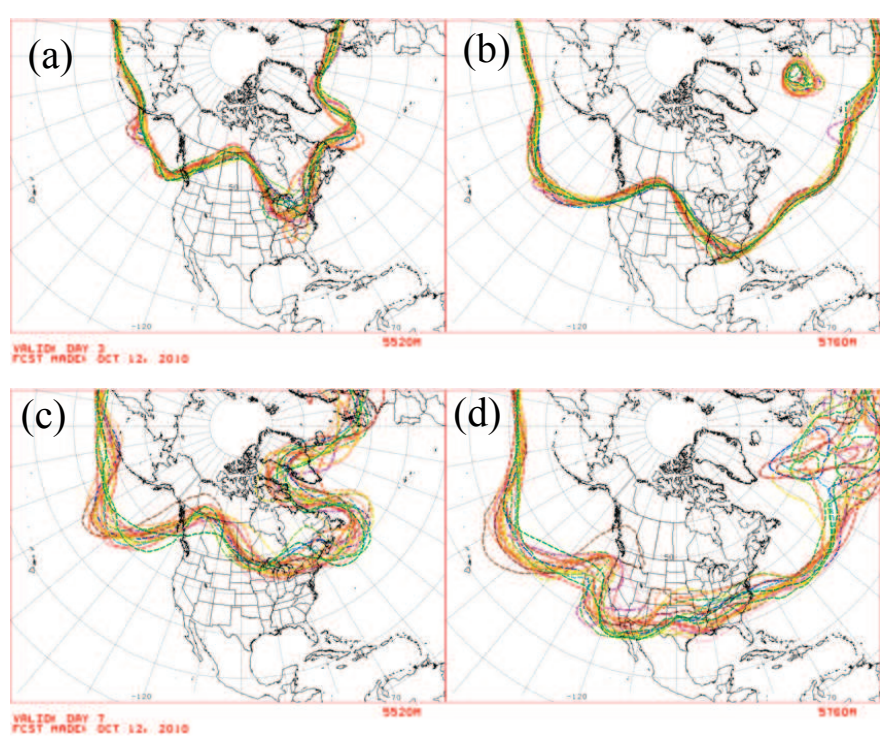

Fig. 3. Ensemble forecasts. Shown are contours of the $500 \mathrm{hPa}$ geopotential height over North America for forecasts started on Oct. 12, 2010. The top panels show the predicted values after 3 days and the bottom panels, after 7 days.

while diffusing outward, there are still several sources of uncertainty. Some of these include the initial population density and measurement of model parameters. Thus in our simulation we consider both an ensemble of models distinguished by slightly different parameter values and an ensemble of slightly different initial conditions assigned to each of these models.

\section{Data Assimilation}

In this section we derive the Local Ensemble Transform Kalman Filter (LETKF) data assimilation procedure authored by Hunt et al. [11]. This method is used to update an ensemble of initial conditions in light of new observations. The formulation uses elements from $[7,11]$. The general approach may be stated as follows: Given an imperfect forecast model which advances a model trajectory from time $t_{n-1}$ to $t_{n}$, $\mathbf{u}_{t_{n}}=\mathbf{F}\left(\mathbf{u}_{t_{n-1}}, t_{n-1}\right)$, and noise-corrupted observational data, $\mathbf{y}_{1}^{o}, \mathbf{y}_{2}^{o}, \ldots, \mathbf{y}_{n}^{o}$, estimate solution trajectory, $\mathbf{u}(t)$, that best fits the observations. Assume now that at each time $t_{i}, i \in 1 \ldots n$, the observation is related to the system state, $\mathbf{u}\left(t_{i}\right)$, by $\mathbf{y}_{i}=\mathbf{H}_{i}\left(\mathbf{u}\left(t_{i}\right)\right)+\varepsilon_{i}$, where $\varepsilon_{i}$ is a Gaussian random vector with mean $\mathbf{0}$ and covariance matrix, $\mathbf{R}_{i}$. Then the problem can be stated precisely by seeking the maximum likelihood estimator of the trajectory that best fits the observational data. In other words, we wish to maximize likelihood function 


$$
L[\mathbf{u}(t)]=\prod_{j=1}^{n} \exp \left(-\frac{1}{2}\left[\mathbf{y}_{j}^{o}-\mathbf{H}_{j}\left(\mathbf{u}\left(t_{j}\right)\right)\right]^{T} \mathbf{R}_{j}^{-1}\left[\mathbf{y}_{j}^{o}-\mathbf{H}_{j}\left(\mathbf{u}\left(t_{j}\right)\right)\right]\right) .
$$

Taking the log of (10) we see that its maximizer corresponds to minimization of the cost function defined by,

$$
J[\mathbf{u}(t)]=\sum_{j=1}^{n}\left[\mathbf{y}_{j}^{o}-\mathbf{H}_{j}\left(\mathbf{u}\left(t_{j}\right)\right)\right]^{T} \mathbf{R}_{j}^{-1}\left[\mathbf{y}_{j}^{o}-\mathbf{H}_{j}\left(\mathbf{u}\left(t_{j}\right)\right)\right] .
$$

When the forecast model and observation operator are nonlinear, cost function $J$ may not have a unique minimizer, and even if it does, finding it can be computationally difficult. The LETKF combats this problem by approximating the minimum in a manner based on the Kalman filter $[12,13]$. We proceed to derive the Kalman filter below, which produces the minimizer analytically for the case of a linear model and observation operator under the assumption that the minimizer represents Gaussian distribution.

\subsection{Kalman Filter}

The Kalman filter is an iterative update scheme that minimizes the cost function $J$ when the system state evolves according to a linear model, $\mathbf{u}_{n}=\mathbf{M}_{n} \mathbf{u}_{n-1}$, and the observation operator is also linear,

$$
\mathbf{y}_{i}=\mathbf{H}_{i} \mathbf{u}_{i}+\varepsilon_{i} .
$$

We begin the derivation with some assumptions. Suppose at time $t_{n-1}$ we have the minimizer, $\overline{\mathbf{u}}_{a_{n-1}}=\overline{\mathbf{u}}\left(t_{n-1}\right)$, which we assume represents a Gaussian distribution with an associated covariance matrix $\mathbf{P}_{a_{n-1}}$. This assumption is motivated by the fact that a Gaussian distribution propagates to a Gaussian distribution under a linear model. In the absence of new observations the most likely estimate of the true system state is the background,

$$
\overline{\mathbf{u}}_{b_{n}}=\mathbf{M}_{n} \overline{\mathbf{u}}_{a_{n-1}} .
$$

The background covariance matrix is

$$
\mathbf{P}_{b_{n}}=\mathbf{M}_{n} \mathbf{P}_{a_{n-1}} \mathbf{M}_{n}^{\mathrm{T}}+\mathbf{C}_{n} .
$$

Here $\mathbf{C}_{n}$ is assumed to be positive definite and represents model error. For simplicity we assume that $\mathbf{C}_{n}=0$. If $\mathbf{u}$ is a state vector and $c$ an arbitrary constant, we assume algebraically that the analysis completes the square of the cost function:

$$
\begin{aligned}
& \sum_{j=1}^{n-1}\left[\mathbf{y}_{j}^{o}-\mathbf{H}_{j} \mathbf{M}_{n-1, j} \mathbf{u}\right]^{T} \mathbf{R}_{j}^{-1}\left[\mathbf{y}_{j}^{o}-\mathbf{H}_{j} \mathbf{M}_{n-1, j} \mathbf{u}\right]= \\
& {\left[\mathbf{u}-\overline{\mathbf{u}}_{a_{n-1}}\right]^{T} \mathbf{P}_{a_{n-1}}^{-1}\left[\mathbf{u}-\overline{\mathbf{u}}_{a_{n-1}}\right]+c .}
\end{aligned}
$$

When a new observation vector, $\mathbf{y}_{n}^{o}$, becomes available at time $t_{n}$, a simple induction argument applied to (15) at the new time, shows the updated analysis minimizes the cost, 


$$
\begin{aligned}
J[\mathbf{u}(t)] & =\left[\mathbf{u}-\overline{\mathbf{u}}_{b_{n}}\right]^{\mathrm{T}} \mathbf{P}_{b_{n}}^{-1}\left[\mathbf{u}-\overline{\mathbf{u}}_{b_{n}}\right]+\left[\mathbf{y}_{n}^{o}-\mathbf{H}_{n} \mathbf{u}\right]^{\mathrm{T}} \mathbf{R}_{n}^{-1}\left[\mathbf{y}_{n}^{o}-\mathbf{H}_{n} \mathbf{u}\right] \\
& =\left[\mathbf{u}-\overline{\mathbf{u}}_{a_{n}}\right]^{T} \mathbf{P}_{a_{n}}^{-1}\left[\mathbf{u}-\overline{\mathbf{u}}_{a_{n}}\right]+\tilde{c} .
\end{aligned}
$$

If one thinks of the covariance matrices in (16) as numbers, the effect of updating the analysis is intuitive. For example suppose $\mathbf{P}_{b_{n}}$ is large compared to $\mathbf{R}_{n}$. Then the inverse $\mathbf{P}_{b_{n}}^{-1}$ will be smaller than $\mathbf{R}_{n}^{-1}$. Hence, the cost function, $J$, gives more weight to the observation and the resulting analysis will give more preference to the observations. Figure 1 illustrates the result of this process. The analysis from the previous step (leftmost circle and set of dots) is evolved under the model resulting in propagation of the uncertainty, represented by the spread of the green dots and size of the blue ovals. The Kalman filter algorithm yields a new analysis that has reduced uncertainty. The lines connecting the dots from the background, analysis, and observation represent the fact that the analysis is computed by finding the optimal linear combination of the discrepancy between the background and observation.

To derive the updated analysis $\overline{\mathbf{u}}_{a_{n}}$ we seek a minimum variance, unbiased estimator of the true state, $\mathbf{u}$, that satisfies the recursive update equation,

$$
\overline{\mathbf{u}}_{a_{n}}=\mathbf{K}_{n}^{\prime} \overline{\mathbf{u}}_{b_{n}}+\mathbf{K}_{n} \mathbf{y}_{n}^{o} .
$$

The matrices $\mathbf{K}^{\prime}$ and $\mathbf{K}$ can be thought of as telling us how much we should trust the background and observations. Below all quantities are at timepoint $n$ so we drop this subscript. We first simplify (17) by eliminating the matrix $\mathbf{K}^{\prime}$. To do so consider the estimation error equations,

$$
\begin{aligned}
\overline{\mathbf{u}}_{a} & =\mathbf{u}+\tilde{\mathbf{u}}_{a}, \\
\overline{\mathbf{u}}_{b} & =\mathbf{u}+\tilde{\mathbf{u}}_{b} .
\end{aligned}
$$

Substituting (17) in for $\overline{\mathbf{u}}_{a}$ and solving for $\tilde{\mathbf{u}}_{a}$ yields

$$
\tilde{\mathbf{u}}_{a}=\mathbf{K}^{\prime} \overline{\mathbf{u}}_{b}+\mathbf{K} \mathbf{y}^{o}-\mathbf{u} .
$$

Now substitute (19) for $\overline{\mathbf{u}}_{b}$ and (12) for $\mathbf{y}^{o}$ into (20). This gives, after rearrangement,

$$
\tilde{\mathbf{u}}_{a}=\left[\mathbf{K}^{\prime}+\mathbf{K} \mathbf{y}^{o}-\mathbf{I}\right] \mathbf{u}+\mathbf{K}^{\prime} \tilde{\mathbf{u}}_{b}+\mathbf{K} \varepsilon .
$$

Since we want the updated analysis to be unbiased, we force $\mathrm{E}\left[\tilde{\mathbf{u}}_{a}\right]=0$. Taking the expected value of both sides of (21) we get

$$
\begin{aligned}
0 & =\left[\mathbf{K}^{\prime}+\mathbf{K H}-\mathbf{I}\right] \mathbf{u}+\mathbf{K}^{\prime} \mathrm{E}\left[\tilde{\mathbf{u}}_{b}\right]+\mathbf{K} \mathrm{E}[\boldsymbol{\varepsilon}] \\
& =\left[\mathbf{K}^{\prime}+\mathbf{K H}-\mathbf{I}\right] \mathbf{u},
\end{aligned}
$$

where we have used the fact that $\varepsilon$ is Gaussian with zero mean, and background is unbiased. It follows that,

$$
\mathbf{K}^{\prime}=\mathbf{I}-\mathbf{K H} .
$$

Substituting this into (17) we ge the Kalman filter update equation,

$$
\overline{\mathbf{u}}_{a}=\overline{\mathbf{u}}_{b}+\mathbf{K}\left[\mathbf{y}^{o}-\mathbf{H} \overline{\mathbf{u}}_{b}\right] .
$$


In (25), $\mathbf{K}$ is called the Kalman Gain matrix. It represents the proper linear combination of the discrepancy between the background and observations that minimizes the variance of the updated analysis.

To determine $\mathbf{K}$ we first find a convenient form for the analysis covariance matrix $\mathbf{P}_{a}$, then minimize variance which is defined by the cost function,

$$
J(\mathbf{K})=\operatorname{tr}\left(\mathbf{P}_{a}\right),
$$

where $t r$ stands for the trace of a matrix. Below we assume that the observation and background error are uncorrelated; i.e., $\mathrm{E}\left[\varepsilon \tilde{\mathbf{u}}_{b}^{T}\right]=\mathrm{E}\left[\tilde{\mathbf{u}}_{b} \varepsilon^{T}\right]=0$. Then since $\overline{\mathbf{u}}_{a}$ and $\tilde{\mathbf{u}}_{a}$ differ only by the state vector, $\overline{\mathbf{u}}$, the analysis covariance matrix is given by,

$$
\begin{aligned}
\mathbf{P}_{a}= & \mathrm{E}\left[\tilde{\mathbf{u}}_{a} \tilde{\mathbf{u}}_{a}^{T}\right] \\
= & \mathrm{E}\left\{(\mathbf{I}-\mathbf{K H}) \tilde{\mathbf{u}}_{b}\left[\tilde{\mathbf{u}}_{b}^{T}(\mathbf{I}-\mathbf{K H})^{T}+\varepsilon^{T} \mathbf{K}^{T}\right]+\right. \\
& \left.\mathbf{K} \varepsilon\left[\tilde{\mathbf{u}}_{b}^{T}(\mathbf{I}-\mathbf{K H})^{T} \varepsilon^{T} \mathbf{K}^{T}\right]\right\} .
\end{aligned}
$$

Using standard properties of expected value and the assumption of uncorrelated errors we see that (29) simplifies to,

$$
\begin{aligned}
\mathbf{P}_{a} & =(\mathbf{I}-\mathbf{K H}) \mathrm{E}\left[\tilde{\mathbf{u}}_{b} \tilde{\mathbf{u}}_{b}^{T}\right](\mathbf{I}-\mathbf{K H})^{T}+\mathbf{K} \mathrm{E}\left[\varepsilon \varepsilon^{T}\right] \mathbf{K}^{T} \\
& =(\mathbf{I}-\mathbf{K H}) \mathbf{P}_{b}(\mathbf{I}-\mathbf{K H})^{T}+\mathbf{K} \mathbf{R} \mathbf{K}^{T} .
\end{aligned}
$$

Next let $\mathbf{N}=\mathbf{H P}_{b} \mathbf{H}^{T}+\mathbf{R}$. Expanding (31) we deduce that

$$
\begin{aligned}
\mathbf{P}_{a} & =\mathbf{P}_{b}-\mathbf{K H} \mathbf{P}_{b}+\mathbf{K H P}_{b} \mathbf{H}^{T} \mathbf{K}^{T}-\mathbf{P}_{b} \mathbf{H}^{T} \mathbf{K}^{T}+\mathbf{K R} \mathbf{K}^{T} \\
& =\mathbf{P}_{b}-\mathbf{K H} \mathbf{P}_{b}-\mathbf{P}_{b} \mathbf{H}^{T} \mathbf{K}^{T}+\mathbf{K}\left(\mathbf{H} \mathbf{P}_{b} \mathbf{H}^{T}+\mathbf{R}\right) \mathbf{K}^{T} \\
& =\mathbf{P}_{b}-\mathbf{K H} \mathbf{P}_{b}-\mathbf{P}_{b} \mathbf{H}^{T} \mathbf{K}^{T}+\mathbf{K N} \mathbf{K}^{T} .
\end{aligned}
$$

To derive the minimizer we differentiate (26) with respect to $\mathbf{K}$ and apply the identities

$$
\begin{gathered}
\frac{\partial}{\partial \mathbf{A}}\left(\operatorname{tr}\left[\mathbf{A} \mathbf{B} \mathbf{A}^{T}\right]\right)=2 \mathbf{A B}, \\
\frac{\partial}{\partial \mathbf{A}}(\operatorname{tr}[\mathbf{A B}])=\frac{\partial}{\partial \mathbf{A}}(\operatorname{tr}[\mathbf{B} \mathbf{A}])=\mathbf{B}^{T} .
\end{gathered}
$$

This yields,

$$
\frac{\partial J}{\partial \mathbf{K}}=-2 \mathbf{P}_{b} \mathbf{H}^{T}+2 \mathbf{K N} .
$$

Setting the derivative to zero and solving for $\mathbf{K}$ we find the candidate for $\mathbf{K}$ is

$$
\mathbf{K}=\mathbf{P}_{b} \mathbf{H}^{T} \mathbf{N}^{-1}
$$

To ensure this minimizes (26) we show that the Hessian is positive semi-definite. A second calculation using (35) and (36) shows that, 


$$
\frac{\partial^{2} J}{\partial \mathbf{K}^{2}}=2 \mathbf{N}^{T},
$$

which is positive semi-definite because $\mathbf{N}$ is the sum of two covariance matrices which are positive definite by definition. Note there are many equivalent forms for $\mathbf{K}$ and $\mathbf{P}_{a}$. Here we state the most computationally efficient forms,

$$
\begin{gathered}
\overline{\mathbf{u}}_{a}=\overline{\mathbf{u}}_{b}+\mathbf{P}_{a} \mathbf{H}^{T} \mathbf{R}^{-1}\left[\mathbf{y}^{o}-\mathbf{H} \overline{\mathbf{u}}_{b}\right], \\
\mathbf{P}_{a}=\left(\mathbf{I}+\mathbf{P}_{b} \mathbf{H}^{T} \mathbf{R}^{-1} \mathbf{H}\right)^{-1} \mathbf{P}_{b}, \\
\mathbf{K}=\mathbf{P}_{a} \mathbf{H}^{T} \mathbf{R}^{-1} .
\end{gathered}
$$

In the next section we derive the LETKF which is an extension of the basic Kalman filter.

\subsection{Local Ensemble Transform Kalman Filter}

Extension of the Kalman filter to the nonlinear scenario entails many difficulties. First, the propagation of the analysis covariance under the model is no longer traceable by (14). Second, the equations derived for the analysis mean and covariance matrix must be adapted because of the assumed nonlinearity of $\mathbf{H}$ and $\mathbf{F}$. One approach to this problem that has proven useful in operational meterology is ensemble Kalman filtering [6]. The main technique is to select an ensemble of $k$ trajectories whose covariance is used to approximate, $\mathbf{P}_{a_{n-1}}$. Each ensemble is then advanced under the model to time $t_{n}$ and the resulting background ensemble sample covariance is used to estimate $\mathbf{P}_{b_{n}}$.

Challenges arise in this approach because the size of $k$ is limited (usually less than a few hundred) due to computational resources and is typically smaller than the model resolution, $m$. If the spread of the ensemble sufficiently approximates $\mathbf{P}_{b_{n}}$, one can generate an accurate analysis. On the other hand, if the spread poorly estimates the background covariance, as is the case when the forecast model has more than $k$ Lyapunov exponents, then analysis fails to correct errors in the forecast model.

The LETKF [11] makes use of localization to overcome the challenges related to the size of $k$. The strategy is to perform the analysis at each point individually by forming a local ensemble over a subset of the model domain. The hope is that the dynamics at a given point are captured over the local region and relatively lowdimensional. If so the local ensemble will sufficiently estimate the background uncertainty and subsequently correct the background forecast at each point, thereby yielding an updated global analysis over the entire model grid. In the case of weather models it has been shown that dynamics in local regions can be regarded as relatively low-dimesional in comparison to the global weather dynamics [21].

The LETKF has several advantages. First it is computationally efficient because the analysis in each local region can be done in parallel where the dimensionality of the matrices in the Kalman filter equations (40)-(42) are reduced. Second the LETKF 
uses a convenient basis to perform the optimization contributing significantly to the computational performance of the algorithm. Third the analysis is computed without use of the model equations; thus, the LETKF is model independent, a key feature with regard to GBM where the biology is poorly understood and modeling efforts are in their infancy. Studies have proven the LETKF to be an accurate and efficient data assimilation approach for weather and ocean models when the analysis is updated over sufficiently small intervals $[9,11,27]$.

\section{Notation}

We now derive the LETKF data assimilation procedure. At time $t_{n-1}$ we start with an analysis ensemble consisting of $m$-dimensional model vectors

$$
\left\{\mathbf{u}_{t_{n-1}}^{a(i)}: i=1 \ldots k\right\} \text {. }
$$

For the case of the Eikenberry model each $\mathbf{u}_{t_{n-1}}^{a(i)}$ is comprised of density of the proliferating and migrating cells, chemorepellant, and extracellular matrix at every grid point of the model geometry. The mean is regarded as the best estimate of the most likely state of the system. Each ensemble member is advanced under the model until time $t_{n}$. This yields the background ensemble,

$$
\left\{\mathbf{u}_{t_{n}}^{b(i)}: i=1 \ldots k\right\}
$$

where

$$
\mathbf{u}_{t_{n}}^{b(i)}=\mathbf{F}\left(\mathbf{u}_{t_{n-1}}^{a(i)}, t_{n-1}\right) .
$$

For the remainder of this chapter we will simplify the notation by omitting all time subscripts, which are assumed to be $t_{n}$. The sample background mean and analysis mean are defined by:

$$
\begin{gathered}
\overline{\mathbf{u}}_{b}=k^{-1} \sum_{i=1}^{k} \mathbf{u}^{b(i)} \\
\mathbf{P}_{b}=(k-1)^{-1} \sum_{i=1}^{k}\left(\mathbf{u}^{b(i)}-\overline{\mathbf{u}}_{b}\right)\left(\mathbf{u}^{b(i)}-\overline{\mathbf{u}}_{b}\right)^{T} \\
=(k-1)^{-1} \mathbf{U}^{b}\left(\mathbf{U}^{b}\right)^{T},
\end{gathered}
$$

where the $i$ th column of the $m \times k$ background ensemble perturbation matrix, $\mathbf{U}^{b}$, is given by $\mathbf{u}^{b(i)}-\overline{\mathbf{u}}_{b}$. The problem now is to determine a suitable analysis ensemble, $\left\{\mathbf{u}^{a(i)}: i=1 \ldots k\right\}$, that has the appropriate mean and covariance matrix,

$$
\begin{gathered}
\overline{\mathbf{u}}_{a}=k^{-1} \sum_{i=1}^{k} \mathbf{u}^{a(i)}, \\
\mathbf{P}_{a}=(k-1)^{-1} \sum_{i=1}^{k}\left(\mathbf{u}^{a(i)}-\overline{\mathbf{u}}_{a}\right)\left(\mathbf{u}^{a(i)}-\overline{\mathbf{u}}_{a}\right)^{T} \\
=(k-1)^{-1} \mathbf{U}^{a}\left(\mathbf{U}^{a}\right)^{T} .
\end{gathered}
$$




\section{Cost Function}

Formally the LETKF computes the analysis by approximately minimizing the analogue of (16) adapted to the nonlinear observation operator:

$$
J(\mathbf{u})=\left[\mathbf{u}-\overline{\mathbf{u}}_{b}\right]^{\mathrm{T}} \mathbf{P}_{b}^{-1}\left[\mathbf{u}-\overline{\mathbf{u}}_{b}\right]+\left[\mathbf{y}^{o}-\mathbf{H}(\mathbf{u})\right]^{\mathrm{T}} \mathbf{R}^{-1}\left[\mathbf{y}^{o}-\mathbf{H}(\mathbf{u})\right] .
$$

Difficulties ensue in adapting the Kalman filter equations (40-42) because the columns of $\mathbf{U}^{b}$ sum to zero, which implies $\operatorname{rank}\left(\mathbf{P}_{b}\right)=\operatorname{rank}\left(\mathbf{U}_{b}\right)<k$. It follows then that $\mathbf{P}_{b}^{-1}$ is not generally defined for all model vectors. However, $\mathbf{P}_{b}$ is symmetric, thus oneto-one of its column space, $S$. This space is the same as the column space of $\mathbf{U}_{b}$, i.e, the span of the background ensemble perturbations vectors. Now if we let $\tilde{S}$ denote a general $k$ dimensional space, we can treat $\mathbf{U}_{b}$ as a linear transformation from $\tilde{S}$ onto $S$. Then our strategy is to find the appropriate linear combination of background ensemble perturbations, $\overline{\mathbf{w}}_{a} \in \tilde{S}$, so that $\overline{\mathbf{u}}_{a}=\overline{\mathbf{u}}_{b}+\mathbf{U}_{b} \overline{\mathbf{w}}_{a}$ minimizes (47).

To justify this approach rigorously, suppose $\mathbf{w}$ has a Gaussian distribution with mean $\mathbf{0}$ and covariance matrix, $(k-1)^{-1} \mathbf{I}$. From properties of Gaussian random vectors we know $\mathbf{u}=\overline{\mathbf{u}}_{b}+\mathbf{U}_{b} \mathbf{w}$ is Gaussian with mean $\overline{\mathbf{u}}_{b}$ and covariance given by (44). Then the analogous cost function for $\mathbf{w}$ defined on the space $\tilde{S}$ is

$$
\tilde{J}(\mathbf{w})=(k-1) \mathbf{w}^{T} \mathbf{w}+\left[\mathbf{y}^{o}-\mathbf{H}\left(\overline{\mathbf{u}}_{b}+\mathbf{U}_{b} \mathbf{w}\right)\right]^{\mathrm{T}} \mathbf{R}^{-1}\left[\mathbf{y}^{o}-\mathbf{H}\left(\overline{\mathbf{u}}_{b}+\mathbf{U}_{b} \mathbf{w}\right)\right] .
$$

We prove that if $\overline{\mathbf{w}}_{a}$ minimizes $\tilde{J}$, then $\overline{\mathbf{u}}_{a}=\overline{\mathbf{u}}_{b}+\mathbf{U}_{b} \overline{\mathbf{w}}_{a}$ minimizes $J$. To see this let $\mathbf{P}$ be the orthogonal projection matrix from $\tilde{S}$ onto the subspace spanned by the columns of $\mathbf{U}_{b}$. Then $\mathbf{P}=\mathbf{U}_{b}\left[\mathbf{U}_{b}^{T} \mathbf{U}_{b}\right]^{-1} \mathbf{U}_{b}^{T}$. Next decompose the vector $\mathbf{w}$ as $\mathbf{w}=\mathbf{P w}+(\mathbf{I}-\mathbf{P}) \mathbf{w}$. Substituting this into (48) for $\mathbf{w}$ only in the first term we get,

$$
\begin{aligned}
(k-1) \mathbf{w}^{T} \mathbf{w} & =(k-1) \mathbf{w}^{T}[\mathbf{P} \mathbf{w}+(\mathbf{I}-\mathbf{P}) \mathbf{w}] \\
& =(k-1) \mathbf{w}^{T} \mathbf{P} \mathbf{w}+(k-1)^{-1} \mathbf{w}^{T}(\mathbf{I}-\mathbf{P}) \mathbf{w} .
\end{aligned}
$$

Utilizing $\mathbf{w}=\mathbf{U}_{b}^{-1}\left(\mathbf{u}-\overline{\mathbf{u}}_{b}\right)$ and the formula for $\mathbf{P}$, we have

$$
\begin{aligned}
(k-1) \mathbf{w}^{T} \mathbf{P} \mathbf{w} & =(k-1)\left(\mathbf{U}_{b}^{-1}\left[\mathbf{u}-\overline{\mathbf{u}}_{b}\right]\right)^{T} \mathbf{U}_{b}\left[\mathbf{U}_{b}^{T} \mathbf{U}_{b}\right]^{-1} \mathbf{U}_{b}^{T} \mathbf{U}_{b}^{-1}\left[\mathbf{u}-\overline{\mathbf{u}}_{b}\right] \\
& =(k-1)\left(\mathbf{U}_{b}^{-1}\left[\mathbf{u}-\overline{\mathbf{u}}_{b}\right]\right)^{T} \mathbf{U}_{b}^{-1}\left[\mathbf{u}-\overline{\mathbf{u}}_{b}\right] \\
& =(k-1)\left[\mathbf{u}-\overline{\mathbf{u}}_{b}\right]^{T}\left(\mathbf{U}_{b}^{-1}\right)^{T} \mathbf{U}_{b}^{-1}\left[\mathbf{u}-\overline{\mathbf{u}}_{b}\right] \\
& =(k-1)\left[\mathbf{u}-\overline{\mathbf{u}}_{b}\right]^{T}\left(\left[\mathbf{U}_{b}\left(\mathbf{U}_{b}\right)^{T}\right]^{-1}\right)\left[\mathbf{u}-\overline{\mathbf{u}}_{b}\right] \\
& =\left[\mathbf{u}-\overline{\mathbf{u}}_{b}\right]^{T}\left(\mathbf{P}_{b}\right)^{-1}\left[\mathbf{u}-\overline{\mathbf{u}}_{b}\right] .
\end{aligned}
$$

Combining (50) and (55) in (48) we deduce that

$$
\tilde{J}(\mathbf{w})=(k-1) \mathbf{w}^{T}(\mathbf{I}-\mathbf{P}) \mathbf{w}+J\left(\overline{\mathbf{u}}_{b}+\mathbf{U}_{b} \mathbf{w}\right) .
$$

The first term on the right is the orthogonal projection of $\mathbf{w}$ onto the null space, $N$, of $\mathbf{U}_{b}$; thus, it depends only on the components of $\mathbf{w}$ in the null space. Similarly the second term only depends on the components in the column space, $S$, of $\mathbf{U}_{b}$. It follows that $\overline{\mathbf{w}}_{a}$ minimizes $\tilde{J}$ if and only if it is orthogonal to $N$ and $\overline{\mathbf{u}}_{a}$ minimizes $J$. 


\section{Analysis Mean and Covariance}

We now proceed to derive the updated analysis and covariance matrix by computing an approximate minimizer to $\tilde{J}$ based on the Kalman filter equations. The results are the analogue of Eqs. (40) and (41). To do so we first obtain the linear approximation of the observation operator. This is accomplished by first applying $\mathbf{H}$ to each background trajectory, $\mathbf{u}^{b(i)}$. This produces the $\ell$-dimensional $(\ell \leq m)$ vectors that comprise the background observation ensemble,

$$
\mathbf{y}^{b(i)}=\mathbf{H}\left(\mathbf{u}^{b(i)}\right) .
$$

Here $\ell$ denotes the spatial dimension of the observations. Denote $\overline{\mathbf{y}}_{b}$ as the mean background observation and $\mathbf{Y}_{b}$ the $\ell \times k$ background observation ensemble perturbation matrix whose $i$ th column is $\mathbf{y}^{b(i)}-\overline{\mathbf{y}}_{b}$. We then take the linear approximation,

$$
\mathbf{H}\left(\overline{\mathbf{u}}_{b}+\mathbf{U}_{b} \mathbf{w}\right) \approx \overline{\mathbf{y}}_{b}+\mathbf{Y}_{b} \mathbf{w} .
$$

Replacing $\mathbf{H}$ with the above and using the assumption that the background mean $\overline{\mathbf{w}}_{b}=0$, the analysis mean satisfies the analogue of (25),

$$
\overline{\mathbf{w}}_{a}=\mathbf{K}\left[\mathbf{y}^{o}-\overline{\mathbf{y}}_{b}-\mathbf{Y}_{b} \mathbf{w}\right] .
$$

Applying equations (40) and (41) with $\mathbf{Y}_{b}$ playing the role of $\mathbf{H}$ produces

$$
\begin{aligned}
\overline{\mathbf{w}}_{a} & =\tilde{\mathbf{P}}_{a} \mathbf{Y}_{b}^{T} \mathbf{R}^{-1}\left(\mathbf{y}^{o}-\overline{\mathbf{y}}_{b}\right), \\
\tilde{\mathbf{P}}_{a} & =\left[(k-1) \mathbf{I}+\mathbf{Y}_{b}^{T} \mathbf{R}^{-1} \mathbf{Y}_{b}\right]^{-1} .
\end{aligned}
$$

In model variables the analysis mean and covariance matrix are determined by

$$
\begin{aligned}
\overline{\mathbf{u}}_{a} & =\overline{\mathbf{u}}_{b}+\mathbf{U}_{b} \overline{\mathbf{w}}_{a}, \\
\mathbf{P}_{a} & =\mathbf{U}_{b} \tilde{\mathbf{P}}_{a} \mathbf{U}_{b}^{T} .
\end{aligned}
$$

\section{Analysis Ensemble}

To complete the derivation of the LETKF we determine an analysis ensemble which has the above mean and covariance. Our strategy is to choose a matrix whose columns sum to zero and have the desired covariance, then shift the columns by $\overline{\mathbf{u}}_{a}$ to achieve the mean in (62). Since the LETKF makes use of localization, we also desire the ensemble to continuously depend on the analysis covariance matrix. This ensures that nearby grid points that have similar matrices, $\tilde{P}_{a}$, yield similar analysis ensembles. One choice with the desired properties is $\mathbf{U}_{a}=\mathbf{U}_{b} \mathbf{W}_{a}$, where $\mathbf{W}_{a}$ is the symmetric square root defined by,

$$
(k-1) \tilde{\mathbf{P}}_{a}=\mathbf{W}_{a} \mathbf{W}_{a}^{T} .
$$

Direct calculation from (46) shows that 


$$
\begin{aligned}
\mathbf{P}_{a} & =\mathbf{U}_{b} \tilde{\mathbf{P}}_{a} \mathbf{U}_{b}^{T} \\
& =\mathbf{U}_{b}(k-1)^{-1} \mathbf{W}_{a} \mathbf{W}_{a}^{T} \mathbf{U}_{b}^{T} \\
& =(k-1)^{-1} \mathbf{U}_{a} \mathbf{U}_{a}^{T}
\end{aligned}
$$

which agrees with (63). The final step is to show the columns of $\mathbf{U}_{a}$ sum to zero and then adjust them by the appropriate vector to ensure that (62) holds. This is equivalent to showing $\mathbf{U}_{a} \mathbf{v}=\mathbf{0}$ where $\mathbf{v}$ is the $k$ by 1 vector defined by $\mathbf{v}=(\mathbf{1}, \mathbf{1}, \ldots, \mathbf{1})^{\mathbf{T}}$. To confirm this note that because the columns of $\mathbf{Y}_{b}$ sum to zero we have,

$$
\begin{aligned}
\tilde{\mathbf{P}}_{a}^{-1} \mathbf{v} & =(k-1) \mathbf{v}+\mathbf{Y}_{b}^{-1} \mathbf{R}^{-1} \mathbf{Y}_{b} \mathbf{v} \\
& =(k-1) \mathbf{v} .
\end{aligned}
$$

Thus $\mathbf{v}$ is an eigenvector for $\tilde{\mathbf{P}}_{a}$ with corresponding eigenvalue $(k-1)^{-1}$. This implies $\mathbf{v}$ is also an eigenvector for $\tilde{\mathbf{P}}_{a}$ with eigenvalue $(k-1)$. But then from (64) we have $\mathbf{P}_{a} \mathbf{v}=(k-1)^{-1} \mathbf{W}_{a} \mathbf{W}_{a}^{T} \mathbf{v}$. It follows then that $\mathbf{v}$ is an eigenvector for $\mathbf{W}_{a}$ with eigenvalue 1. From this we see that

$$
\begin{aligned}
\mathbf{U}_{a} \mathbf{v} & =\mathbf{U}_{b} \mathbf{W}_{a} \mathbf{v} \\
& =\mathbf{U}_{b} \mathbf{v} \\
& =0,
\end{aligned}
$$

due to the columns of $\mathbf{U}_{b}$ summing to 0 . To form the analysis we first add $\overline{\mathbf{w}}_{a}$ to each column vector, $\mathbf{w}^{a(i)}$, of $\mathbf{W}_{a}$. Denote the resulting vectors $\tilde{\mathbf{w}}^{a(i)}$ and matrix $\widetilde{\mathbf{W}}_{a}$. Then the $i$ th analysis ensemble member is defined by $\mathbf{u}^{a(i)}=\overline{\mathbf{u}}_{b}+\mathbf{U}_{a} \tilde{\mathbf{w}}^{a(i)}$. The updated analysis mean is,

$$
\begin{aligned}
\overline{\mathbf{u}}_{a} & =k^{-1} \sum_{i=1}^{k}\left(\overline{\mathbf{u}}_{b}+\mathbf{U}_{a} \tilde{\mathbf{w}}^{a(i)}\right) \\
& =\overline{\mathbf{u}}_{b}+\mathbf{U}_{b} \overline{\mathbf{w}}_{a}+k^{-1} \mathbf{U}_{b} \sum_{i=1}^{k} \mathbf{w}^{a(i)} \\
& =\overline{\mathbf{u}}_{b}+\mathbf{U}_{b} \overline{\mathbf{w}}_{a}+k^{-1} \mathbf{U}_{b} \mathbf{W}_{a} \mathbf{v} \\
& =\overline{\mathbf{u}}_{b}+\mathbf{U}_{b} \overline{\mathbf{w}}_{a},
\end{aligned}
$$

as desired.

\subsection{Computational Implementation of the LETKF}

Computation of the analysis mean, covariance and ensemble as derived above is accomplished through the following steps. The LETKF procedure begins with several preliminary calculations carried out over the entire model grid. First the observation operator is applied to the $m$-dimensional background ensemble vectors $\mathbf{u}^{b(i)}$ to form the background observation ensemble $\mathbf{y}^{b(i)}$. Next both ensembles are averaged and the vectors $\mathbf{y}^{b(i)}-\overline{\mathbf{y}}_{b}$ and $\mathbf{u}^{b(i)}-\overline{\mathbf{u}}_{b}$ are computed. These vectors are then used to form the perturbation matrices $\mathbf{U}_{b}$ and $\mathbf{Y}_{b}$. The remaining steps below are performed for each local region. 
1. Select the components of $\mathbf{u}^{b(i)}, \mathbf{y}^{b(i)}, \mathbf{U}_{b}, \mathbf{Y}_{b}$ and $\mathbf{R}$ that correspond to the local region. We denote the resulting local background ensemble and background ensemble perturbation matrix $\mathbf{x}^{b(i)}$ and $\mathbf{X}_{b}$, respectively.

2. Compute the $k \times \ell$ matrix $\mathbf{C}=\mathbf{Y}_{b}^{\mathrm{T}} \mathbf{R}^{-1}$. (If the observations are not independent and $\mathbf{R}$ is not diagonal, it is computationally more efficient to solve the system $\mathbf{R C}^{\mathrm{T}}=\mathbf{Y}_{b}$ instead of inverting $\mathbf{R}$.)

3. Compute the $k \times k$ symmetric matrix $\tilde{\mathbf{P}}_{a}=\left[(k-1) \mathbf{I} / \rho+\mathbf{C Y}_{b}\right]^{-1}$. (See below for more discussion of $\rho$.)

4. Compute the $k \times k$ matrix $\mathbf{W}_{a}=\left[(k-1) \tilde{\mathbf{P}}_{a}\right]^{1 / 2}$. This choice ensures that $W_{a}$ depends continuously on the elements of $\tilde{\mathbf{P}}_{a}$. (Otherwise, small changes in $\tilde{\mathbf{P}}_{a}$ at neighboring grid points can lead to very different analysis ensembles $[11,31]$.)

5. Compute the $k$-vector $\overline{\mathbf{w}}_{a}=\tilde{\mathbf{P}}_{a} \mathbf{C}\left(\mathbf{y}^{o}-\overline{\mathbf{y}}_{b}\right)$ and add it to each column of $\mathbf{W}_{a}$ to form the $k \times k$ analysis weight matrix $\widetilde{\mathbf{W}}_{a}$.

6. Compute the analysis perturbation matrix $\mathbf{X}_{a}=\mathbf{X}_{b} \widetilde{\mathbf{W}}_{a}$.

7. The analysis ensemble, $\mathbf{x}^{a(i)}$, is formed by adding $\overline{\mathbf{x}}_{b}$ to the $i$ th column of $\mathbf{X}_{a}$, $i=1,2, \ldots, k$.

The data assimilation process is completed by forming the global analysis ensemble, $\mathbf{u}^{a(i)}$, which consists of the collection of local analysis ensembles, $\mathbf{x}^{a(i)}$, at the center of each local region. In principle, the only free parameters in the LETKF scheme are the ensemble size, $k$, and the size of each local region. In practice, however, the model is always an imperfect representation of the underlying dynamics. As a result, ensemble methods tend to underestimate the actual background uncertainty, which causes them to underweight the observations when the new analysis is computed. In severe cases, the filter can diverge. One ad hoc remedy is to "inflate" the background ensemble covariance by a tunable parameter. The procedure described above has the effect of multiplying the background ensemble perturbations by $\sqrt{\rho}$, thereby helping to ensure the analysis gives appropriate weight to the observations.

\section{Results}

In meteorology, tests of proposed data assimilation systems are called observing system simulation experiments (OSSEs). Because the weather is a complex multiscale process, one hopes to separate the effects of observation density and error from model error. In a perfect model simulation, one creates a "truth run" from a fixed initial condition with the same model that is used to make the ensemble forecasts. At intervals, synthetic noisy observations are generated from the "truth." The goal of the OSSE is to determine how well a forecast ensemble tracks the truth when the synthetic observations are assimilated using a forecast model that is identical to the model used for the truth run. Such experiments can quantify the effect of noise and observation density and frequency on the accuracy of the analyses, since there is no model error.

Contrary to weather prediction, where the models are well developed, efforts to forecast a true patient GBM are likely to have significant sources of model error 
because current models, such as the ones used here, represent crude idealizations of the true tumor dynamics. Thus our numerical experiments explore a range of sources of model error which are likely to be found in the clinical setting. While a model with a given set of parameter values may reasonably predict the growth of a tumor, the underlying heterogenous and genetically unstable cell population may cause unaccounted for perturbations in key growth and migration rates. Additionally, GBM patients typically experience a combination of treatments including surgery, chemotherapy, and radiotherapy whose effects on the tumor are not well understood or considered here.

Given the above anticipated sources of error, we establish proof of concept for the data assimilation method in the GBM setting. This is accomplished by adapting the LETKF procedure to the two models of glioblastoma discussed in this chapter while accounting for several sources of uncertainty. We start with a "truth" tumor whose dynamics are assumed to be governed exactly by the Eikenberry model, Eqs. (3-6), with parameter values given by table 2 . An ensemble of 25 tumors is evolved under the Logistic Swanson model, Eq. (2). Tumor heterogeniety and error due to parameter estimation is approximated by assigning each ensemble tumor a unique logistic growth rate $(\alpha)$, carrying capacity $\left(T_{\max }\right)$ and diffusion rate in white matter $\left(D_{w}\right)$ (the preferred path of migration [24]), from a uniformly distributed random variable in the intervals reported in table 3 . The parameter values are assigned at initialization and remain fixed over the entire simulation. (In reality parameters are likely to vary in time). All other parameter values for the ensemble tumors are identical to those reported in table 1 . The truth and ensemble tumors are initialized by integrating each under their respective models until they reach an approximate diameter between 15 $\mathrm{mm}$ and $20 \mathrm{~mm}$, measured over grid points with cell density over 80 percent of carrying capacity.

The range of parameters values in table 3 are chosen for several reasons. First because the models used here exhibit simple dynamics, where the cell population generally grows to carrying capacity and diffuses outward, it is necessary numerically to vary parameters such as the carrying capacity over the ensemble. This prevents the background covariance matrix from becoming ill-conditioned and its inverse in (47) tending to infinity which can cause the filter to diverge or neglect the observations. Second, as our results will show, we wish to demonstrate the ability of the LETKF to better estimate the true tumor state even in the presence of significant model parameter error. For example, we vary $D_{w}$ in table 3 by several orders of magnitude. The result is a set of tumors that vary greatly in degree of invasion. This is clinically relevant because diffusion rates may vary greatly on a patient by patient basis resulting in substantial uncertainty in the tumor growth.

Our OSSE then begins with generation of a synthetic observation of the truth and the LETKF data assimilation procedure performed using a local region size of $7 \mathrm{~mm}$ by $7 \mathrm{~mm}$. The choice of region size is motivated by the empirical assessment that the areas of greatest forecast uncertainty are along the edges of the tumor, meaning the boundary of the enhancing region. This yields an updated analysis after which the truth and ensemble are integrated for 30 days, and the process is repeated for 6 
cycles totaling 180 days worth of simulation. The process is finite due to the fatal nature of GBM tumors.

Table 3. Ensemble parameter value ranges

\begin{tabular}{ll}
\hline Parameter & Range of values \\
\hline$\alpha$ & {$[0.15,0.35]$} \\
$T_{\max }$ & {$[8000,12000]$} \\
$D_{w}$ & {$\left[6.5 \times 10^{-4}, .16\right]$} \\
\hline
\end{tabular}

In our simulations the observation operator, $\mathbf{H}(x)$, represents the MRI that would be observed if the tumor state were $\mathbf{x}$. In our initial experiment we regard the tumor state to be the cell density at each point on the model geometry. As previously discussed in the introduction, many details about the relationship between the tumor state and the contrast enahncement are not well characterized, and there is intrinsic variability arising from uptake of the contrast agent and other aspects of MR image generation such as interrater reliability. For our purposes we assume the enhancement varies linearly with the tumor cell density at each point on the domain, up to a random error.

The value of $\mathbf{H}$ is computed pointwise as follows. Let $u_{k}(\mathbf{x}, t)$ be the tumor cell density for the $k$ th ensemble member at location $\mathbf{x}$ and time $t$. Then

$$
h_{k}(\mathbf{x})=\max \left(0, \min \left(1, \frac{u_{k}(\mathbf{x}, t)}{T_{\max }^{k}}+\eta(\mathbf{x})\right)\right),
$$

where $\eta(\mathbf{x})$ is a uniformly distributed random value in the interval $[-0.1,0.1]$ and $T_{\max }^{k}$ is the carrying capacity for the $k$ th ensemble solution. The value of $h_{k}$ (which is confined to the unit interval) is the component of the $\mathbf{H}$ corresponding to the location $\mathbf{x}$ in the brain domain. Note that the $\eta$ 's are independent. The synthetic observations are computed by applying $\mathbf{H}$ to the truth with the appropriate carrying capacity.

Figure 4 shows the results of our first experiment. Each plot displays the cell density where above 5 cells $\mathrm{mm}^{-2}$ and the brain geometry below. The color coding is done on a 128 color linear scaling analogous to a temperature plot where red corresponds to cells at or near the carrying capacity of the truth and blue represents lower cell densities. The first, second, and third rows correspond to days 1, 90, and 180 of the experiment. The left column, labeled "background," shows the background ensemble mean. The middle column, labeled "analysis," shows the analysis mean. The right column, labeled "truth," displays the truth. We see that each time the data assimilation is performed, the resulting analysis has improved tracking of the truth.

Figure 5 displays the level curves for a five member subset of the ensemble. Two level curves are shown for each ensemble tumor corresponding to 25 and 80 percent of truth carrying capacity. The left and right columns display the background and analysis ensemble, respectively. The decrease in the spread of the level curves following data assimilation illustrates a reduction in uncertainty in estimated size and 


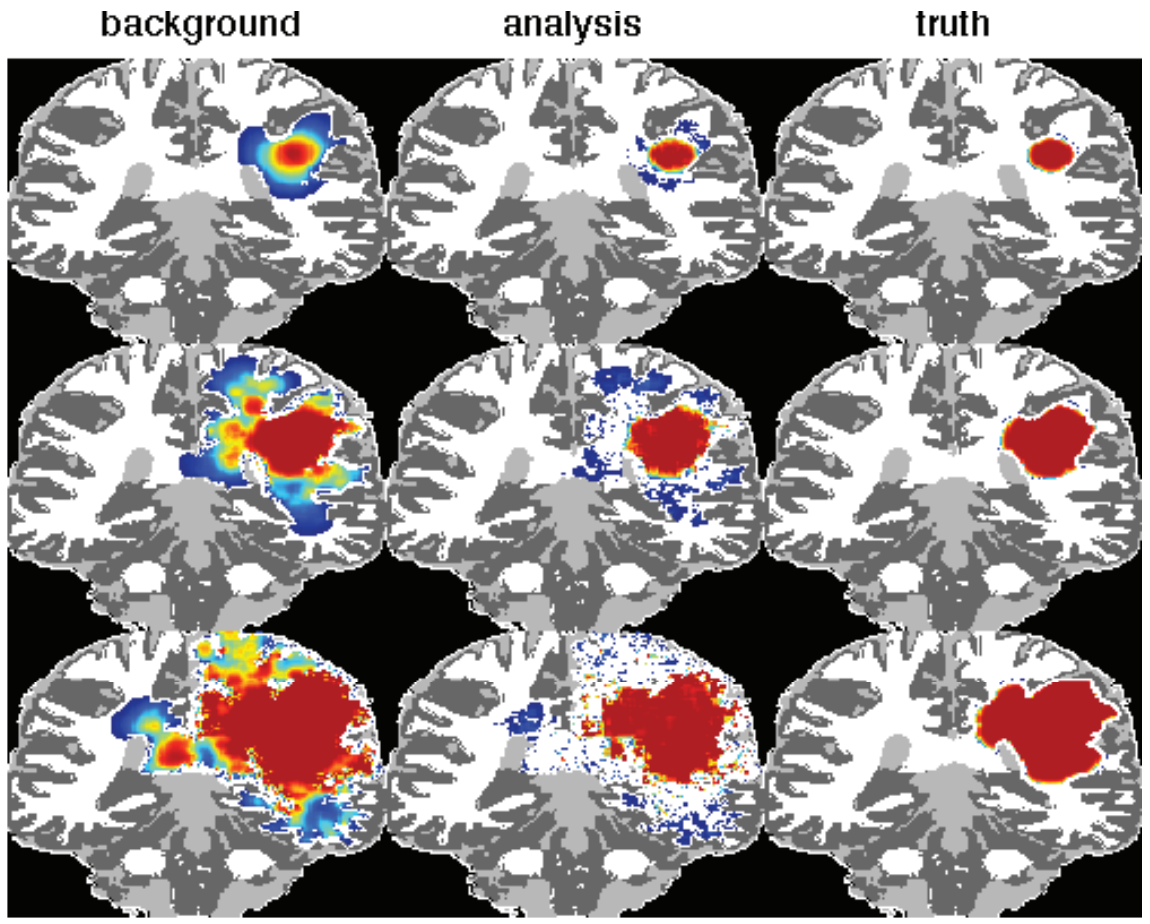

Fig. 4. Cell density plots for our first observing system simulation experiment. The first, second, and third rows correspond to day 1,90, and 180 of the experiment. See text for additional details.

location of the truth tumor. In clinical practice this would be useful in assessing the location of the tumor boundary and the overall size of the tumor.

For comparison we also conducted a free run of the experiment where data assimilation is not performed and the ensemble is simply integrated for 180 days under their respective models. Figure 6 displays the tumor mass every tenth day of the experiment. The blue curve shows the ensemble mean tumor mass for the simulation where data assimilation is performed. The error bars corresponding to one standard deviation evaluated over the ensemble are also displayed. The red curve displays the tumor mass for the truth. The green curve shows the ensemble mean tumor mass for the free run. Note how data assimilation yields a forecast that more accurately estimates the truth tumor mass. Every 30th day when the LETKF is applied we see a reduction in uncertainty in the estimated tumor mass and well as improved quantitative agreement with the truth.

We perform a second experiment where we assumed the enhancement level is linearly related to the time derivative of the growing cell population (proliferating cells for solutions to Eikenberry model). That is, regions of highest enhancement correspond to the areas with the greatest cell growth rate. The formulation for $h_{k}$ in this case is derived from the fact that solutions to the logistic equation have max- 


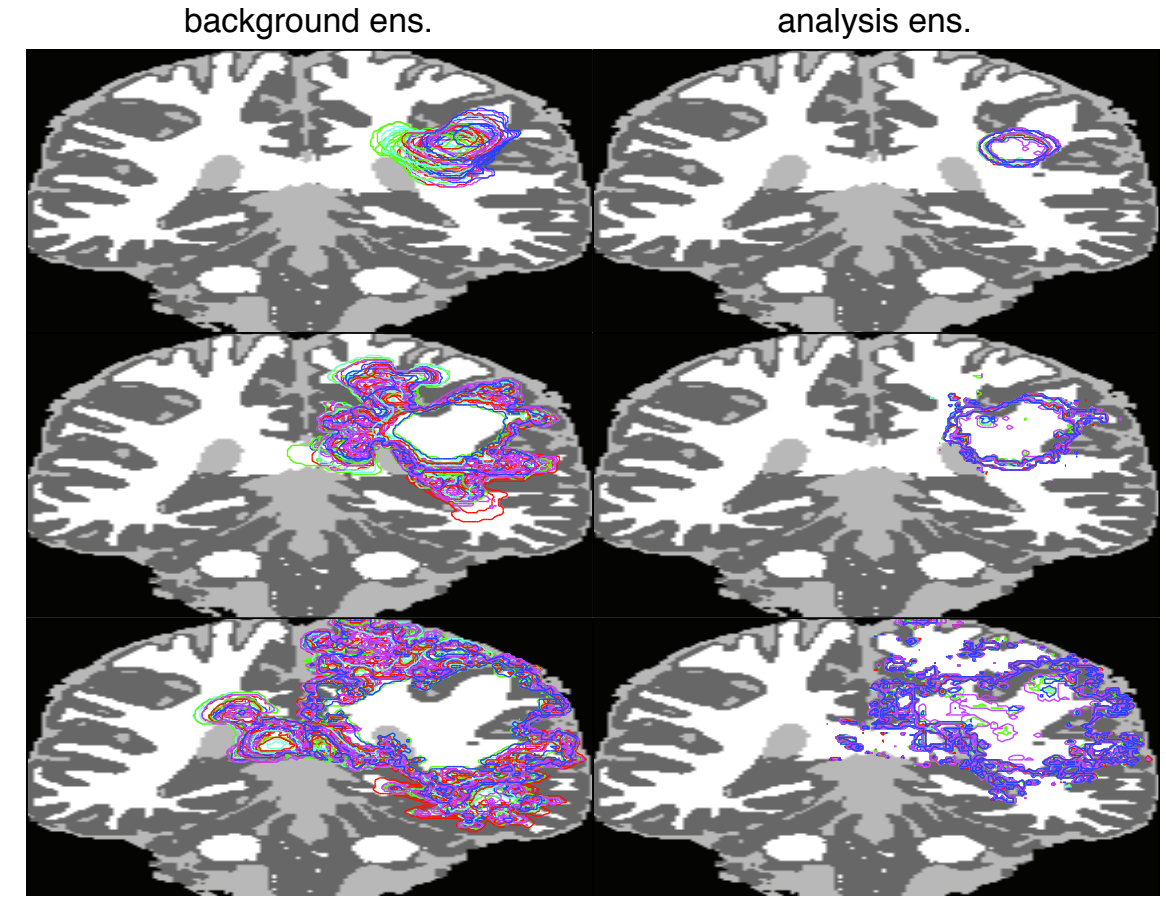

Fig. 5. Contour plot for a subset of the ensemble tumors. See text for additional details.

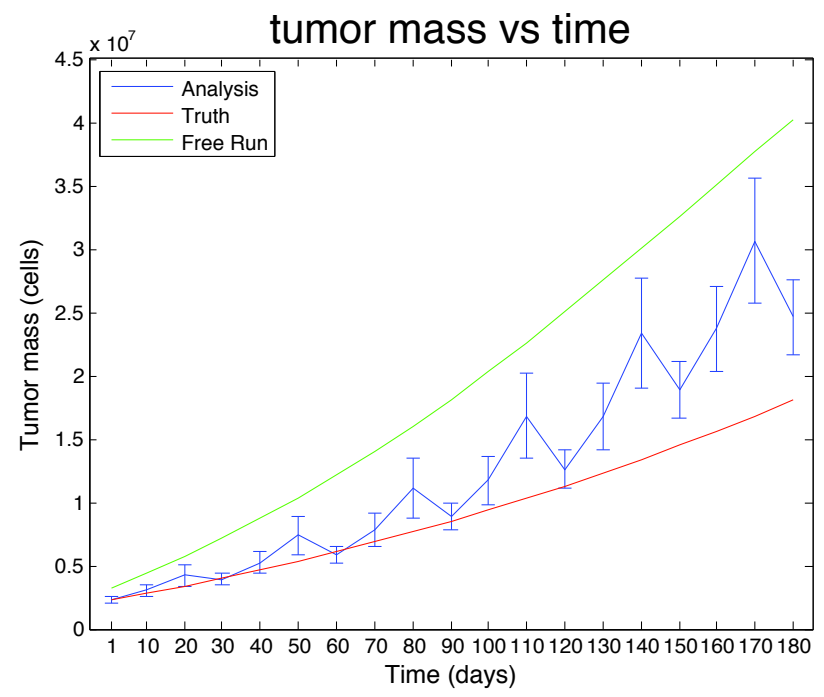

Fig. 6. Plot of tumor mass vs time for first observing system simulation experiment. See text for additional details. 
imal derivative at half carrying capacity. Thus to find the upper bound on the time derivative of the growing cells we simply evaluate the right hand side of the logistic equation, $g \prime=\alpha g\left(1-\frac{g}{T_{\max }}\right)$, at $g=\frac{T_{\max }}{2}$. The upper bound on the derivative is $\frac{\alpha T_{\max }}{4}$. Hence the analogue of $(65)$ is

$$
h_{k}(\mathbf{x})=\max \left(0, \min \left(1, \frac{4 \frac{\partial u_{k}(\mathbf{x}, t)}{\partial t}}{\alpha_{k} T_{\max }^{k}}\right)+\eta(\mathbf{x})\right)
$$

where $\alpha_{k}$ is the logistic growth rate of the $k$ th ensemble solution and $\frac{\partial u_{k}(\mathbf{x}, t)}{\partial t}$ is the time derivative of the growing cell density for the $k$ th ensemble member. $\eta(x)$ is as in (65).

Figure 7 displays results of the experiment with (66) used to form the components of the observation operator. The rows correspond to days 120,150, and 180 of the experiment. In this case assimilation of observations yields an analysis that has invaded essentially the same area of the truth, but the tumor core (region composed red grid points) is heterogenous and slightly smaller in overall size. If we compare the analysis on day 120 to the background at day 150 , we see that the analysis grows to have about the same tumor core size as the truth between data assimilation steps, but has a greater area of infiltration. Taking into consideration that for the chosen parameter values, the ensemble mean is more invasive than the truth and that the contrast enhancement for this case depends on the time rate of change of the growing cells, we can think of the data assimilation as having a "braking" effect that temporarily slows down the overall growth of the ensemble mean.

For this experiment we assess forecast error in terms of the root-mean-square (RMS) difference of the total tumor cell population:

$$
\operatorname{rmse}\left(\bar{T}, t_{n}\right)=\left(V^{-1} \sum_{\mathbf{x}}\left[T_{\text {true }}\left(\mathbf{x}, t_{n}\right)-\bar{T}\left(\mathbf{x}, t_{n}\right)\right]^{2}\right)^{1 / 2},
$$

where $\bar{T}$ gives the tumor cell density at each point of the analysis ensemble mean and the sum is over every point of the computational domain (whose 2-dimensional volume is $V$ ). Figure 8 displays the RMS vs time plot for this experiment (blue curve) and the free run where no data assimilation is performed (red curve). The error bars on the blue curve display one standard deviation in the RMS evaluated over the ensemble. Observe how application of the LETKF yields an ensemble forecast that has better overall accuracy than the free run of the experiment.

We conducted similar experiments to those presented here where the range of parameter values is changed as well as simulations where the ensemble and truth evolve according to the same model. The overall results were similar in all experiments to what we have reported in this chapter.

\section{Effect of Varying Observation Frequency}

We explore the effect of varying the period between assimilation steps. To do so our first experiment is repeated with synthetic observations generated every one, seven, 


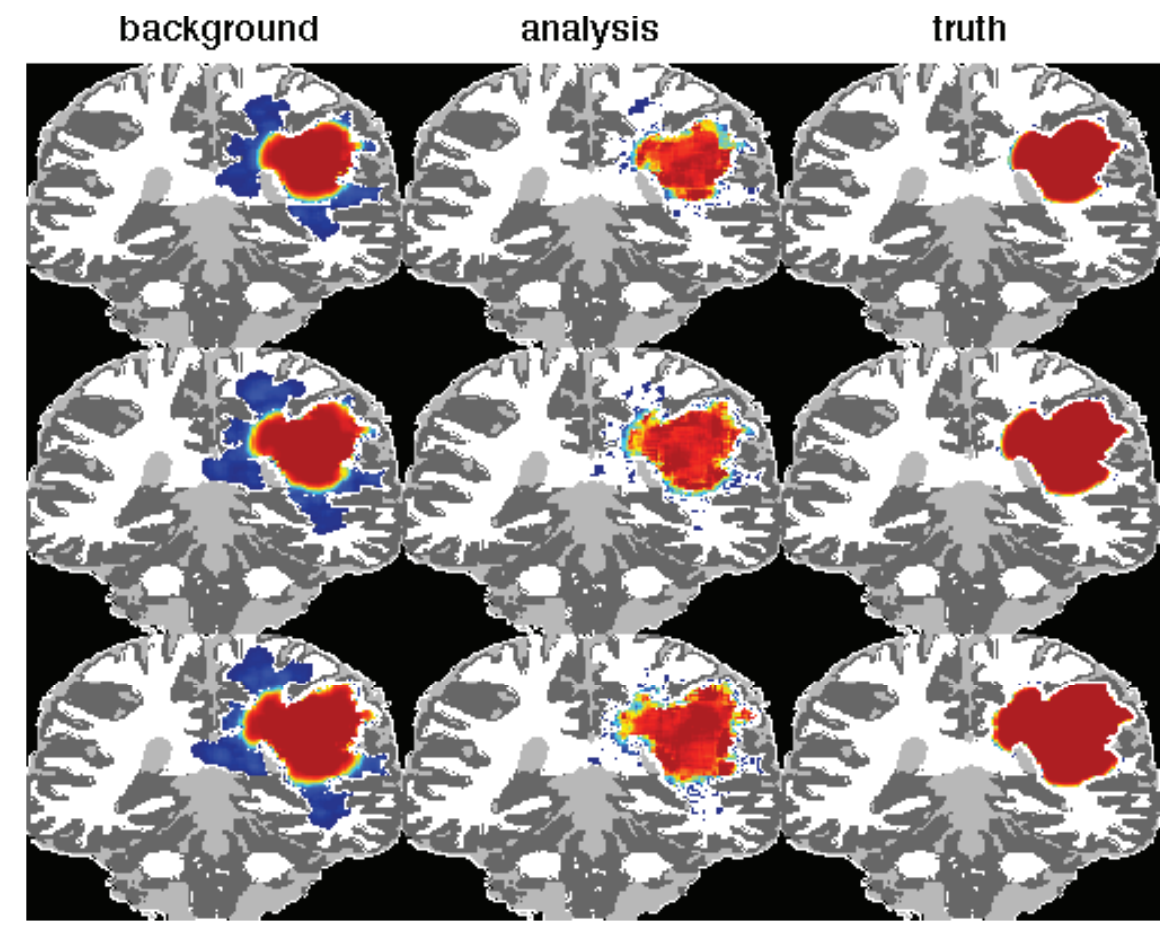

Fig. 7. Cell density plots for the second observing system simulation experiment where (66) is used to form the components of the observation operator. The first, second, and third rows correspond to day 120,150 , and 180 of the experiment.

30, 60, 90, and 180 day(s). Each simulation is still conducted over a period of 180 days beginning and ending with a data assimilation cycle. Figure 9 shows the final analysis mean cell density. The subplot titled "Daily" corresponds to observations everyday. "Weekly" corresponds to observations every 7 days. The other subplots are titled analogously. In every case but the quarterly run, the final ensemble mean gives a good approximation of the truth tumor core (region in red) with varying degrees of satellitosis. The edema (blue region) surrounding the main tumor mass varies significantly over the experiments and in some cases gives a poor approximation to the truth (see the truth in figure 4). This is because when observations are available frequently, for example in the daily run, the errors in the model do not sufficiently propagate between assimilation cycles. The resulting background ensemble has little variance in the edema region causing the updated analysis to essentially ignore the observations locally. Despite varying the frequency of observations, the simulations show that the LETKF data assimilation scheme can still provide an improved approximation to the true tumor state. This is clinically relevant because usually patient images are collected at irregular intervals and typically only at initial diagnosis and before and after surgery. 


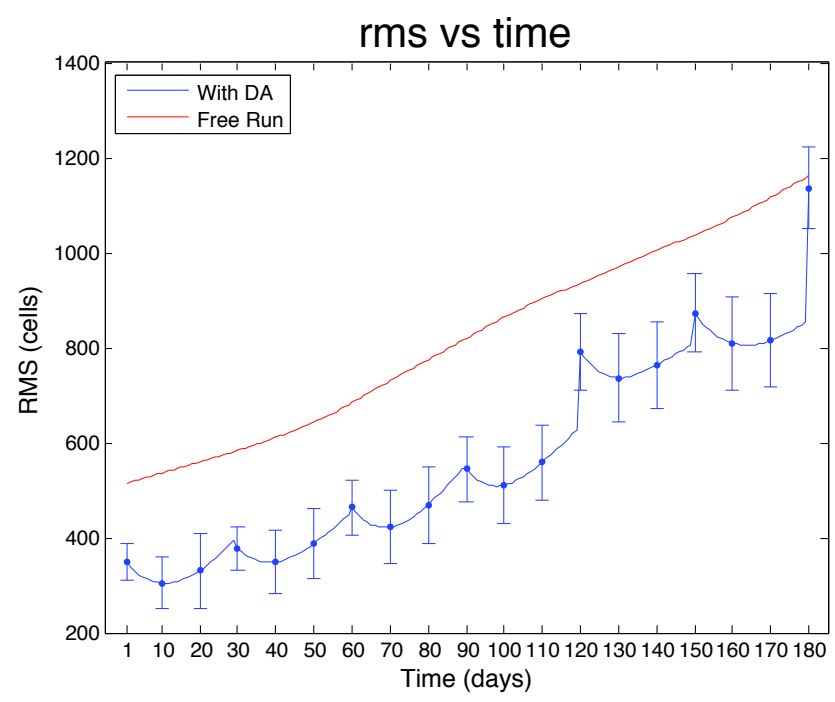

Fig. 8. Plot of the rms vs time for every tenth day of the second presented experiment. See text for additional details.

\section{Conclusion and Discussion}

The results of this chapter demonstrate the use of ensemble forecasting and data assimilation to make improved estimates of future growth of a simulated glioblastoma given synthetically generated observations of the tumor. The two experiments presented explore different models of the relationship between the tumor state and the contrast enhancement in an MR image. In both cases the ensemble mean had improved tracking of the truth when data assimilation is performed despite the substantial sources of model and observational error. This demonstrates the potential feasibility of this framework for use in human cases of glioblastoma with real patient MR image data.

Several related problems must be considered before the data assimilation approach can be used as a clinical aid. First the effect of surgery, radiotherapy, and chemotherapy on the glioma cell population needs to be incorporated into the forecast model. Similarly any ensemble forecast must account for the uncertainty and variance in key treatment parameters such as the size of the surgical resection cavity, treatment dosage and timing, the application field for radiotherapy or chemotherapy, and development of resistance by glioma cells.

A third problem concerns the use of patient imaging data in an ensemble forecast. Here we have modeled the relationship in an ad hoc way where we assume the pixel intensity directly relates to the tumor state under the statistical assumption of uncorrelated errors that have a Gaussian distribution. In reality it is difficult to model the relationship between cell density and pixel intensity due to the complex means by which tumors evolve. Additionally there are many factors previously discussed 


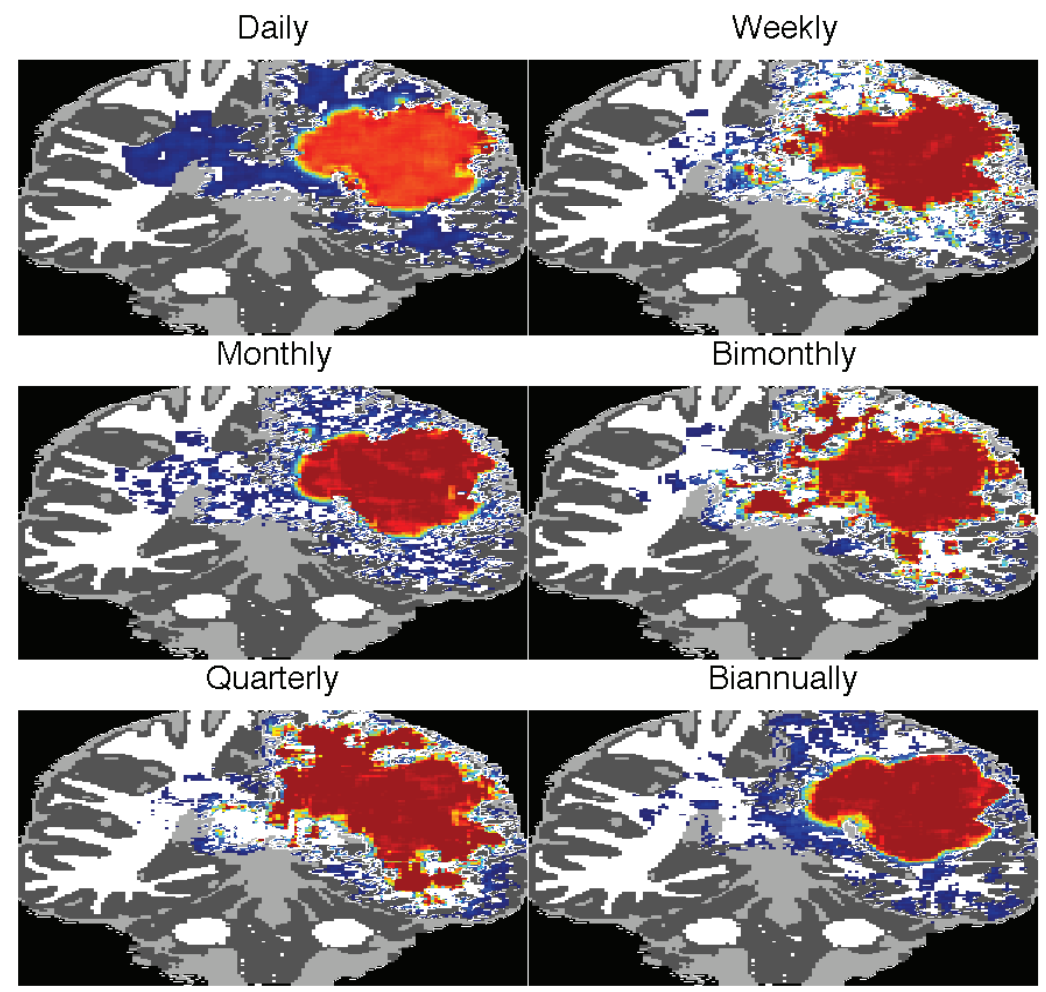

Fig. 9. Final cell density plots for the first experiment repeated where the time period between observations is varied. See text for details.

that lead to uncertainty in a patient tumor MR image. Also any assumed observation operator will likely have non-Gaussian distributed error with covariance matrix that is difficult to estimate.

Future work will focus on refinement of the forecast model and validation of this approach with real clinical imaging data. Our ultimate goal is to apply data assimilation methods as a treatment aid to help improve management of patient GMB tumors and positively affect their quality of life.

Acknowledgements. Portions of this work were funded by the Barrow Neurological Institute Women's Foundation and by funds from the Newsome Family Endowed Chair of Neurosurgery Research held by Dr. Preul. J.M. was supported in part by an Achievement Reward for College Scientists Scholarship. Y.K. was supported by NSF grants DMS-0436341 and DMS-0920744. 


\section{References}

1. Amberger, V.R., Hensel, T., Ogata, T.N., and Schwab, M.E.: Spreading and migration of human glioma and rat C6 cells on central nervous system myelin in vitro is correlated with tumor malignancy and involves a metalloproteolytic activity. Cancer Res., 58, 149-158 (1998)

2. http://www.bic.mni.mcgill.ca/brainweb/.

3. Clatz, O., Sermesant, M., Bondiau, P.-Y., Delingette, H., Warfield, S.K., Malandian, G., and Ayache, N.: Realistic simulation of the $3 \mathrm{~d}$ growth of brain tumors in MR images coupling diffusion with biomechanical deformation. IEEE Trans. Med. Imaging, 24, 1334 1346 (2005)

4. Demuth, T. and Berens, M.E.: Molecular mechanisms of glioma cell migration and invasion. J. Neurooncol., 70, 217-228 (2004)

5. Eikenberry, S.E., Sankar, T., Preul, M.C., Kostelich, E.J., Thalhauser, C.J., and Kuang, Y.: Virtual glioblastoma: growth, migration and treatment in a three-dimensional mathematical model. Cell Prolif., 42, 511-528 (2009)

6. Evensen, G.:Data Assimilation: The Ensemble Kalman Filter, Springer (2006)

7. Gelb, A. (ed): Applied Optimal State Estimation. MIT Press, Cambridge, Ma., (1974)

8. Grossman, A., Helbich, T.H., Kuriyama, N., Ostrowitzki, S., Roberts, T. P., Shames, D.M., van Bruggen, N., Wendland, M.F., Israel, M.A., and Brasch, R.C.: Dynamic contrastenhanced magnetic resonance imaging as a surrogate marker of tumor response to antiangiogenic therapy in a xenograft model of glioblastoma multiforme. J. Magn. Reson. Imaging, 15, 233-240 (2002)

9. Hoffman, R.N, Ponte, R.M., Kostelich, E.J., Blumberg, A., Szunyogh, I., Vinogradov, S.V., and Henderson, J.M.: A simulation study using a local ensemble transform Kalman filter for data assimilation in New York Harbor. J. Atmos. Ocean Tech., 25, 1638-1656 (2008)

10. Horton, J.R.: An Introduction to dynamic meteorology. 4th ed. Amsterdam: Elsevier Academic Press (2004)

11. Hunt, B.R., Kostelich, E.J., and Szunyogh, I.: Efficient data assimilation for spatiotemporal chaos: A local ensemble transform Kalman filter. Physica D, 230, 112-126 (2007)

12. Kalman, R.E.: A new approach to linear filtering and prediction problems. Trans. ASME Ser. D: J. Basic Eng., 82, 35-45 (1960)

13. Kalman, R.E., and Bucy, R.S.: New results in linear filtering and prediction theory. Trans. ASME Ser. D: J. Basic Eng., 83, 95-108 (1961)

14. Kalnay, E.: Atmospheric modeling, data assimilation, and Predictability. Cambridge University Press (2003)

15. Lorenz, E.N.: Deterministic non-periodic flow. J. Atmos. Sci., 20, 130-141 (1963)

16. Lorenz, E.N.: A study of the predictability of a 28 -variable atmospheric model. Tellus, 17, 321-333 (1965)

17. Marino, S., Hogue, I.B., Ray, C.J., and Kirschner, D.E.: A methodology for performing global uncertainty and sensitivity analysis in systems biology. J. Theor. Biol., 254, 178196 (2008)

18. Medical Image Processing, Analysis, and Visualization, available from the Center for Information Technology, National Institutes of Health, Bethesda, MD. See mipav.cit.nih.gov

19. Mohamed A., and Davatzikos, C.: Finite element modeling of brain tumor mass-effect from 3D medical images. In: Duncan J.S., Gerig, G. (eds) 8th International Conference on Medical Image Computing and Computer Assisted Intervention(MICCAI). Springer, Palm Springs CA 400-408 (2005) 
20. Norden, A.D., and Wen, P.Y.: Glioma therapy in adults. Neurologist. 12, 279-292 (2006)

21. Patil, D.J., Hunt, B.R., Kalnay, E., Yorke, J.A., and Ott E.: Local low dimensionality of atmospheric dynamics. Phys. Rev. Lett., 86, 5878-5881 (2001)

22. M. Rijpkema, J.H. Kaanders, F.B. Joosten, A.J. van der Kogel, and Heerschap, A.: Method for quantitative mapping of dynamic MRI contrast agent uptake in human tumors. $J$. Magn. Reson. Imaging, 14, 457-463 (2001)

23. Stein A.M., Demuth T., Mobley D., Berens M., and Sander L.: A mathematical model of glioblastoma tumor spheroid invasion in a $3 \mathrm{D}$ in vitro experiment. Biophys. J., 92, 356-365 (2007)

24. Swanson, K.R., Alvord, Jr., E.C., and Murray, J.D.: A quantitative model of differential motility of gliomas in white and grey matter. Cell Prolif., 33, 317-329 (2000)

25. Swanson, K.R., Bridge C., Murray, J.D., and Alvord, E.C.: Virtual and real brain tumors: using mathematical modeling to quantify glioma growth and invasion. J. Neurol. Sci., 216, 1-10 (2003)

26. Swanson, K.R., Rostomily, R.C., and Alvord, Jr., E.C.: A mathematical modeling tool for predicting survival of individual patients following resection of glioblastoma: A proof of principle. Brit. J. Cancer, 98, 113-119 (2008)

27. Szunyogh, I., Kostelich, E.J., Gyarmati, G., Kalnay, E., Hunt, B.R., Ott, E., Satterfield, E., and Yorke, J.A.: A local ensemble Kalman filter data assimilation system for the NCEP global model. Tellus A, 60, 113-130 (2008)

28. Talairach, J. and Tournoux, P.: Co-Planar Stereotaxic Atlas of the Human Brain: 3-D Proportional System: An Approach to Cerebral Imaging. Thieme Medical Publishers, New York (1988)

29. Tian, J.P., Friedman, A., Wang, J., and Chiocca, E.A.: Modeling the effects of resection, radiation and chemotherapy. J. Neurooncol., 91, 287-293 (2009)

30. Vose, M.D.: The Simple Genetic Algorithm: Foundations and Theory. The MIT Press, Cambridge, MA (1999)

31. Wang, X., Bishop, C.H., and Julier, S.J.: Which is better, an ensemble of positive negative pairs or a centered spherical simplex ensemble?. Mon. Wea. Rev., 132, 1590-1605 (2004)

32. Wasserman R., Acharya R., Claudio S., and Shin K.H.: A patient-specific specic in vivo tumor model. Math. Biosci., 136, 111-140 (1996) 